\title{
LA NEGOCIACIÓN COLECTIVA ANTE LOS RIESGOS LABORALES EN LA NUEVA ERA DIGITAL
}

\section{Collective negotiation in the face of occupational risks in the digital age}

\author{
M. a Belén Fernández Collados \\ Universidad de Murcia, España
}

\begin{abstract}
RESUMEN
La nueva era digital ha supuesto grandes cambios en el sistema económico y productivo que comportan nuevos riesgos para la salud de los trabajadores y trabajadoras. Este ensayo, que forma parte del resultado de una investigación más extensa sobre el impacto de la Industria 4.0 en el trabajo ${ }^{1}$, pretende analizar el papel que desempeña y puede llegar a desempeñar la negociación colectiva en la prevención de tales riesgos. A tal fin, antes de examinar los instrumentos negociales, es preciso sentar ciertas premisas sobre el Derecho del Trabajo en la que ha sido calificada como industria 4.0, así como considerar cuáles son los riesgos laborales y las medidas de prevención, para finalmente llegar a una serie de conclusiones y propuestas.
\end{abstract}

Palabras clave: prevención, negociación colectiva, salud, tecnología, digitalización.

\section{ABSTRACT}

The new digital era has meant great changes in the economic and productive system that entail new risks to the health of workers. This essay, which is part of the result of a more extensive research on the impact of Industry 4.0 at work, aims to analyze the role that collective negotiation plays and can play in the prevention of such risks. To this end, before examining the collective negotiation, it is necessary to establish certain premises about Labor Law in which it has been classified as Industry 4.0, as well as to consider what are the occupational risks and prevention measures, to finally reach a series of conclusions and proposals.

Keywords: prevention, Collective negotiation, health, technology, digitization.

1 Esta obra queda enmarcada dentro de los trabajos de investigación desarrollados por la autora en el Proyecto financiado por la Comunidad Autónoma de la Región de Murcia a través de la convocatoria de Ayudas a proyectos para el desarrollo de investigación científica y técnica por grupos competitivos, incluida en el Programa Regional de Fomento de la Investigación Científica y Técnica (Plan de Actuación 2018) de la Fundación Séneca-Agencia de Ciencia y Tecnología de la Región de Murcia: 20976/PI/18: El impacto de la Industria 4.0 en el trabajo: Una visión interdisciplinar.

\footnotetext{
* Correspondencia a: María Belén Fernández Collados. C/ Cuesta del Olivar, n. ${ }^{0}$ 54, 30430 Cehegín (Murcia), España - mbelenfc@ um.es - https://orcid.org/0000-0002-8294-1554

Cómo citar: Fernández Collados, María Belén. (2020). «La negociación colectiva ante los riesgos laborales en la nueva era digital»; Lan Harremanak, 44, 56-78. (https://doi.org/10.1387/lan-harremanak.22053).
}

Recibido: 22 septiembre, 2020; aceptado: 09 octubre, 2020.

ISSN 1575-7048 - elSSN 2444-5819 / (C) 2020 UPV/EHU 


\section{Aspectos introductorios sobre el Derecho del Trabajo en la era digital}

La evolución del Derecho del Trabajo históricamente ha sido coetánea a los grandes cambios tecnológicos, que a lo largo de los tiempos han planteado dos cuestiones básicas: la suplantación de las personas por máquinas y nuevos riesgos para la salud.

Si por algo se distingue la que podría denominarse como nueva era digital, industria 4.0, o cuarta revolución industrial, es porque a diferencia de las clásicas revoluciones industriales, ésta va más allá de la introducción de un mero cambio tecnológico en la producción industrial, no significa sin más el paso del uso de un tipo de energía o tecnología a otro en una industria de características similares a la de la época fordista. La digitalización industrial no consiste exclusivamente en la incorporación al proceso industrial de tecnologías como: Big data; Blockchain o cadena de bloqueo; Cloud computing o computación en la nube; Fabricación aditiva o fabricación por adición; Impresión 3D; Inteligencia artificial; Realidad aumentada; Realidad virtual; Ciberseguridad o seguridad en la red; 5G; Robots y robots colaborativos; las redes sociales; y el denominado Internet de las cosas (IoT)... Además, no puede contextualizarse en un único y exclusivo modelo de producción, ni siquiera en un solo modelo de economía, por ello, en términos genéricos, tampoco se puede hablar de una solución global o unívoca aplicable a las nuevas contingencias derivadas de la industria 4.0 en general.

La era digital se caracteriza por la compra-venta on line, la sustitución de la inteligencia humana por el Iot, la domótica, la robótica, e incluso por el big data — si de lo que se trata es de analizar grandes datos- Las plataformas digitales están consolidándose en todos los países como una nueva forma de negocio. La «on-demand economy» está cada vez más presente y las rental platforms, craft platforms o financing platforms y gig platforms forman parte ya desde hace unos años de nuestro modelo económico y social. Se ha normalizado el hecho de acudir a páginas webs o apps para obtener cualquier tipo de servicio. De hecho, son precisamente los sectores de actividad más tradicionales (restauración, viajes, televisión, transporte...) los que prestan sus servicios a través de plataformas digitales como Uber, Airbnb, Booking.com, TripAdvisor, BlaBlaCar, Glovo, HBO, Netflix, Amazon..., de tal manera que las startups, al igual que la economía colaborativa, o el crowdsourcing, han pasado a formar parte de la cotidianeidad en la oferta y la demanda de gran parte de los servicios prestados a la comunidad.

En líneas generales, la digitalización industrial comporta la irrupción de un nuevo modelo de organización, la incorporación de sistemas automatizados con los que lograr una producción más eficiente, la reducción de los trabajos manuales a la par que el aumento de los trabajos de dirección y gestión, la flexibilización del horario e incluso del lugar de trabajo como consecuencia del uso de las 
nuevas tecnologías de la información y de la comunicación, una mayor interacción directa con el cliente y transparencia en los procesos. Es más, un elemento común a estas modernas formas de producción y prestación de servicios del siglo xxI es la posibilidad de favorecer la interconexión de personas y cosas, generando un importante volumen y flujo de datos para su agrupamiento, almacenamiento y análisis (Torrecilla García et al., 2019: 30 y ss.).

Como consecuencia de todo ello, y de los propios cambios tecnológicos, vuelve a replantearse como ya ocurriera en cada una de las llamadas revoluciones industriales, además de la aparición de nuevas enfermedades del trabajo, la contundente pérdida de empleos -fundamentalmente en los trabajos menos cualificados-, a lo que se une un nuevo temor: la mayor precarización laboral como consecuencia de la cuasi-desaparición del trabajo dependiente y el crecimiento de los contratos a demanda frente al contrato indefinido.

Con todo, debe partirse de la premisa de que «La tecnología puede destruir empleos, pero no trabajo» (Ruesga, 2019: 259), siendo un hecho incontestable que de los cambios tecnológicos también surgen nuevas oportunidades de negocio hasta hace poco insospechadas, siendo un claro ejemplo en esta nueva era digital, entre otros muchos, el de las youtubers e influencers.

La prestación de servicios a través de páginas webs o apps, la también denominada economía de plataformas, puede clasificarse en tres modalidades: economía de plataformas puramente colaborativa; economía de plataformas bajo demanda, que a su vez se puede subdividir en crowdwok online y crowdwork offline (Todoli Signes, 2017:21); y economía de plataformas de acceso (Martín Muñoz, 2020). En cualquiera de ellas no sólo se ve afectada la relación entre empresa y cliente, también el modelo tradicional de relación entre empresa y trabajador/a, siendo muy difícil de encajar en el concepto clásico de Derecho del Trabajo (Hernández Bejarano, 2016) y en la tradicional distinción entre trabajo autónomo y dependiente, fundamentalmente en las plataformas puramente colaborativas como BlaBlacar (García-Perrote Escartín et al., 2017).

La platform economy genera microworkers cuyas notas características podrían concretarse en tres (Mercader Uguina, 2017: 613): 1) organización y atribución de tareas a través de algoritmos; 2) trasparencia; 3) y ¿cierta autonomía y no exclusividad?. Las dos primeras notas no repercuten en la laboralidad de la prestación del servicio, pues nada obsta a que la planificación y la asignación de tareas se realice a través de un algoritmo capaz de elaborarla más eficientemente, mediante la asignación de las tareas al profesional que en cada momento concreto reúna los requerimientos profesionales y geográficos más apropiados para cubrir las necesidades del cliente y que todo el procedimiento de la prestación de servicios quede registrado, garantizando una transparencia que genera un alto nivel de confianza entre los proveedores de servicios y quienes los reciben. Así pues, es en la autonomía de quienes prestan los servicios donde reside el cuestiona- 
miento sobre la laboralidad de la prestación. Aunque depende de la plataforma concreta, puesto que aún con rasgos comunes, cada forma de negocio presenta ciertas singularidades, siendo básico poder determinar si quien presta los servicios puede o no rechazar la tarea encomendada y si tiene o no exclusividad con la empresa.

Los Tribunales se han pronunciado en EE.UU. y en Reino Unido declarando la laboralidad de la prestación de servicios para Úber, entre otros, en el Caso O'Connor v. Uber Technologies de 11 de marzo de 2015 del Tribunal de Distrito de los Estados Unidos para el Distrito del Norte de California y en la sentencia del Employment Tribunal of London 26 de octubre de 2016, Aslam v. Uber, ampliamente tratados por la doctrina científica nacional (Gutiérrez Arranz, 2016 y Ginés i Fabrellas et al., 2016). Y en España, los Tribunales Superiores de Justicia han estimado la laboralidad de la prestación de servicios, de los denominados riders de empresas como Deliveroo [STSJ de Madrid (Sala de lo Social, Sección 1.a) de 17 enero de 2020 (AS 2020, 534)] y Glovo [STSJ de Asturias (Sala de lo Social, Sección 1. a) de 25 julio de 2019 (AS 2019, 1826)].

El carácter laboral de las nuevas relaciones en el seno de la industria 4.0 repercute directamente en el objeto de este estudio en el que se enlaza prevención de riesgos laborales y negociación colectiva. Por un lado, porque a los riesgos laborales derivados del uso de las nuevas tecnologías digitales se suman los relacionados con estas nuevas fórmulas de producción, organización y gestión empresarial, y, por otro lado, porque según se trate o no de una prestación de servicios encuadrable en el art. 1 del Real Decreto Legislativo 2/2015, de 23 de octubre, por el que se aprueba el texto refundido de la Ley del Estatuto de los Trabajadores (en adelante, ET), no sólo se verán condicionadas las obligaciones en materia de prevención de riesgos laborales, sino que, asimismo, tendrán cabida o no un tipo u otro de instrumentos negociales. En este sentido, debe advertirse que, pese a que con base en el artículo 15 de la Constitución española tanto el Estatuto de los Trabajadores como el Estatuto del Trabajador Autónomo (Ley 20/2007, de 11 de julio, del Estatuto del trabajador autónomo, LETA en adelante), reconocen el derecho a la protección de los trabajadores y establecen un marco normativo aplicable en materia preventiva, éste es muy diferente en ambos supuestos y comporta significativas diferencias en el nivel de protección (Rodríguez Egío, 2018: 504-505). Por otra parte, mientras que a las prestaciones laborales se les aplicaría el convenio colectivo, si bien, lo cuestionable es determinar el convenio que les resulta de aplicación, dada la baja probabilidad de que las plataformas digitales negocien un convenio colectivo de empresa, a las relaciones laborales autónomas económicamente dependientes, les serían de aplicación los acuerdos de interés profesional, como los suscritos por la empresa Deliveroo con la Asociación Española de Rider Mensajero (ASORiders) en 2018 y con la Asociación Autónoma de Riders (AAR) en 2019, pero ¿qué ocurre con las prestaciones de servicios por trabajadores/as autónomos/as?. 
Una parte de la doctrina científica sostiene que actualmente la normativa laboral y de seguridad social nacional carece de un marco regulador suficiente donde encuadrar las nuevas formas de trabajo y la incipiente incorporación de la inteligencia artificial propia de la era digital (Sierra Benítez, 2015: 93-118) y se debate entre quienes estiman que la solución pasa bien por la creación de un nuevo y específico marco jurídico para estas actividades (Mercader Uguina, 2017: 114), bien por la adaptación del existente a través de una relación laboral especial atendiendo a sus particularidades (Todolí Signes, 2017: 76)), e incluso entre quienes entienden que no es preciso un nuevo marco jurídico, ni una relación especial, sino que basta con adaptar los elementos que configuran la relación jurídico laboral a estas nuevas realidades (Jover Ramírez, 2018).

En cualquier caso, en plena era digital el carácter tuitivo del Derecho del Trabajo debe continuar prevaleciendo y el papel de la autonomía colectiva sigue siendo clave en la regulación del trabajo y en la prevención de los riesgos laborales. Si bien, la negociación colectiva tiene un difícil encaje en el seno de la gigeconomy, al debatirse la dependencia en la prestación de los servicios, y en determinados casos hasta la ajeneidad o la retribución. Igualmente, la digitalización afecta directamente a la deslocalización productiva e influye de manera contundente en la representación sindical y por ende en la negociación colectiva, dado que sigue siendo un reto del Derecho del Trabajo español que se tome en consideración como factor de regulación individual y sindical el lugar de trabajo o el trabajo sin lugar, y se abandone el centro de trabajo como eje hegemónico del sistema jurídico (Lahera Fortaleza, 2019: 24-25).

No obstante, «el grado de digitalización de las empresas españolas es muy deficitario, tanto en términos de implantación, uso y aprovechamiento de las $\mathrm{TIC}^{2}$, como de inversión en $\mathrm{I}+\mathrm{D}+\mathrm{i}$ » (Varela Ferrío, 2019: 30), una situación que se ha puesto de manifiesto durante el confinamiento por la crisis de la $\mathrm{CO}$ VID-19. En Espańa, las empresas caracterizadas por la fusión de las tecnologías de la comunicación, el uso de internet y de las nuevas tecnologías no han sido reemplazadas por la industria digitalizada, que se está implantando en sectores muy determinados, de manera que ambas modalidades conviven y no parece que, por lo menos en un corto espacio temporal, vaya a ser sustituido un modelo de producción por otro.

En definitiva, esta investigación parte de las siguientes premisas: que la industria digital no sólo conlleva el empleo de nuevas tecnologías en la producción, también un cambio en el modelo de organización y gestión empresarial que tiene una clara influencia en la salud de los trabajadores y trabajadoras; que no hay un patrón único de producción característico de la era digital, lo que

2 Tecnologías de la Información y la Comunicación. NTIC: nuevas tecnologías de la información y la comunicación. 
complica sobremanera el tratamiento de la seguridad y salud laboral en la industria 4.0 en términos genéricos; que una de las características de la gig-economy es su pretendida huida del Derecho del Trabajo; que la negociación colectiva ha de salvar ciertas dificultades para regular el trabajo en la era digital; y que la digitalización de la industrial española es mucho más lenta que la de otros países ${ }^{3}$. Y una vez sentadas tales premisas como base de la investigación, el siguiente objetivo es analizar los riesgos laborales derivados de la digitalización industrial y cuál está siendo el papel de la negociación colectiva, con el fin de poder extraer conclusiones y hacer propuestas ad futurum que puedan contribuir a la mejora de la seguridad y salud de los trabajadores y trabajadoras.

\section{Los riesgos laborales derivados de la digitalización industrial y las posibles medidas de prevención}

La aplicación de las NTIC en el ámbito laboral reporta grandes beneficios al sistema productivo, entre otros: facilita el trabajo, favorece niveles de productividad y eficiencia impensables en otros tiempos..., pero, como todo, también presenta "otra cara", que se traduce en nuevos retos para la salud y el bienestar en el trabajo (Fernández Avilés, 2017: 73 y ss.).

Por todos es conocido que la transformación de los sistemas productivos lleva aparejada la evolución también de los riesgos laborales, de manera tal que los riesgos emergentes pasan a ser una nueva realidad e irrumpen otros hasta la fecha desconocidos.

A grandes rasgos, podría decirse que en la industria del siglo XIX los riesgos laborales estaban más focalizados en las condiciones de higiene, que fueron mejorando con una considerable regresión de las enfermedades profesionales. También en el siglo xx, la mecanización de los procesos industriales y la incorporación de maquinaria más segura, supuso una importante optimización de las condiciones de seguridad y la disminución de los accidentes de trabajo. De manera tal, que desde finales del siglo xx y principios del xxI, aun sin descuidar la higiene y la seguridad industrial, el foco de atención se centra en los riesgos ergonómicos y psicosociales, ligados al uso de las tecnologías de la información y la comunicación, a las nuevas formas de organización del trabajo y la digitalización de la producción industrial y el intercambio de bienes y servicios.

Los trabajadores y trabajadoras de los entornos vinculados a la digitalización industrial están expuestos a nuevas tecnologías que conllevan riesgos práctica-

\footnotetext{
${ }^{3}$ En Espańa el porcentaje de empresas que utilizan la inteligencia artificial está muy por debajo de la media europea según el informe Ipsos para la Comunidad Europea.

https://www.elcomercio.es/tecnologia/inteligencia-artificial-empresas-20200803073103-ntrc.html
} 
mente desconocidos, como son los derivados de la impresión 3D, la implementación de la nanotecnología, la fabricación de semiconductores y otras técnicas novedosas. No obstante, como ya se ha señalado, en plena era digital, la ergonomía y psicosociología del trabajo adquieren un papel fundamental. Si bien, dichos riesgos no provienen en sí de la incorporación de las nuevas tecnologías a las relaciones laborales, sino de la inadecuada gestión y uso de esas tecnologías por parte de la organización empresarial (González Cobadela, 2019: 88).

Aunque como se indicó, en el siglo xxI no puede hablarse de un modelo único de producción o de empresa, sí que de forma muy genérica pueden delimitarse algunos de esos riesgos — básicamente psicosociales- asociados al uso de las nuevas tecnologías propias de la era digital.

Con carácter general, los factores de riesgo imperantes en la era digital son los referentes a las condiciones y gestión del trabajo, los aspectos organizacionales, los derivados de las nuevas formas de trabajo asociadas al ework, las plataformas digitales, el trabajo crowdsourcing (González Cobaleda, 2019: 97 y ss.)... E incluso, el hecho de que en los modernos modelos de organización empresarial los empleados y empleadas se vean más implicados en la toma de decisiones y asuman nuevas responsabilidades y nuevas dinámicas requeridas por las interfaces humano-máquina (Torrecilla García et al., 2019: 47).

Por un lado, como ya aconteció con otros cambios tecnológicos, el empleo de los dispositivos móviles y de las NTIC, junto a los riesgos físicos (molestias oculares y problemas de visión, fatiga muscular, inflamaciones de articulaciones y tendones...), conllevan otros riesgos de carácter psíquico, derivados del esfuerzo en la adaptación a los nuevos métodos y herramientas de trabajo, de la sustitución de determinados puestos de trabajo por aplicaciones informáticas, así como de la obsolescencia de las capacidades formativas en un entorno laboral en constante evolución. Todo ello sin obviar los que podrían calificarse de riesgos «tradicionales» o la reconversión de esos riesgos clásicos conforme a las nuevas formas de producción.

Por otro lado, la prestación de servicios a través de páginas webs o apps, el teletrabajo, la deslocalización del lugar de trabajo o la consolidación de la empresa "panóptica» (Mercader Uguina, 2018) ${ }^{4}$, entre otros factores, acarrean una constante disponibilidad sin una clara delimitación entre la vida personal y laboral, un ritmo de trabajo difícilmente controlable, la prolongación de la jornada de trabajo, la multitarea propia del empleo de las nuevas tecnologías y su apre-

4 Término acuñado por Mercader Uguina que se refiere al «control a través de sistemas de videovigilancia, microfónicos y telefónicos, el rastreo a través de sistemas de geolocalización; los controles biométricos; el control informático de los niveles de productividad de los trabajadores en tiempo real; el seguimiento de los correos electrónicos y de las navegaciones por internet; el impacto de las redes sociales o, en fin, la enorme proyección que sobre lo laboral comienzan a tener las técnicas del Big Data». 
miante necesidad de respuesta, así como un aumento de la dependencia del uso de esos dispositivos, a lo que ha de sumarse el alto grado de exigencia que impone un sistema productivo en el que la actividad humana es cada vez más indispensable (Meléndez Morillo-Velarde, 2016).

El uso de las NTIC está muy relacionado con la adicción al trabajo (workalcoholic), caracterizada por una excesiva dedicación al mismo, hasta el punto de convertirlo en el único objetivo vital, que, junto a la nomofobia, o miedo irracional a estar sin teléfono móvil o a estar desconectado, constituyen algunos de los riesgos laborales más en auge en el ámbito de la gig-economy, fundamentalmente en el caso de los denominados e-nómadas, que por las características de organización y gestión de su actividad laboral y su desarrollo en espacios virtuales, quedan sometidos a una constante disponibilidad y conectividad, lo que conduce a que la tecnología haya «invadido su vida profesional y personal con un horario de trabajo que no tiene hora de fin establecida", borrándose totalmente la línea que separa la vida personal y profesional (Álvarez Cuesta, 2017).

Los tradicionales riesgos psicosociales como el desgaste profesional o síndrome del quemado (burnout), la violencia en el trabajo o el acoso laboral, adquieren nuevos tintes en la era digital. Así pues, el impacto del acoso puede ser muy superior a través de las redes sociales y el ciberacoso, y en el ejercicio de la violencia en el trabajo se amplían los posibles sujetos activos, por ejemplo, en el caso de las plataformas digitales de trabajos a demanda, ésta puede llegar a ser ejercida por los propios clientes, de los que depende la valoración del trabajo realizado, que repercute en la asignación de otras tareas y/o de la retribución.

En el seno de la industria 4.0 el estrés laboral muta en el denominado tecnoestrés ${ }^{5}$ y sus derivados: tecnoansiedad, tecnofatiga y tecnoadicción, se erigen como el principal riesgo psicosocial proveniente de la digitalización laboral. La tecnoansiedad supone el rechazo o actitud negativa hacia las nuevas tecnologías, entre otras razones, por la falta de adaptación a las mismas, mientras que la tecnofatiga, no deriva necesariamente del rechazo hacia las NTIC, sino del cansancio mental por su uso continuado, sin perjuicio de que pueda verse afectado también por cierto escepticismo sobre su eficacia. Por el contrario, la tecnoadicción es propiciada por el exceso en el empleo de las NTIC que desemboca en una imperante necesidad y en la pérdida de control en su uso (Fernández Martínez, 2019).

Estas fórmulas de tecnoestrés están estrechamente vinculadas a la alteración de los ritmos de trabajo como consecuencia del empleo de las NTIC. Con las NTIC se aceleran los tiempos de recepción de información y respuesta, favore-

5 El concepto de tecnoestrés proviene del psiquiatra norteamericano Craig Brod, quien, en 1984, en su libro Technostress: The Human Cost of the Computer Revolution, se refiere a él como «una enfermedad de adaptación causada por la falta de habilidad para tratar con las nuevas tecnologías del ordenador de manera saludable». 
ciendo la «infobesidad» o «infoxicación». El exceso de información proveniente de diferentes fuentes digitales es, además, en muchos casos irrelevante, complicándose la tarea de filtrarla y analizarla para tomar decisiones de manera eficiente (Fernández Avilés, 2017: 74).

Circunscribiéndonos exclusivamente a la prestación de servicios a través de las plataformas digitales de trabajo a demanda, se han señalado como principales riesgos laborales (Mella Méndez, 2020):

— La inseguridad jurídica derivada de la anomia laboral propia de las plataformas y que supone una evidente fuente de conflictos entre la plataforma y el trabajador, respecto a la naturaleza jurídica de su vínculo, y entre la plataforma y los empresarios tradicionales del sector en el que opera haciéndoles competencia (sirva de ejemplo el conflicto entre Uber y el sector tradicional del taxi).

- La inseguridad personal y laboral intrínseca a la fisionomía del trabajo en las plataformas digitales (no se contratan trabajadores sino prestaciones de servicios, lo que supone una búsqueda permanente de tareas, la necesidad de compatibilizar diferentes trabajos y la competencia entre trabajadores por la asignación de tareas).

- Los derivados del empleo de las NTIC, tanto de carácter psíquico (tecnoadicción, tecnofobia, tecnoansiedad...), como de carácter físico (trastornos oculares, musculoesqueléticos...).

- La precariedad de las condiciones laborales, asumiendo el propio trabajador los costes de ejecución del trabajo e incluso las condiciones de seguridad y salud laboral.

Las medidas de prevención para luchar contra los riesgos laborales propios de la industria 4.0 son tan diversas como los propios riesgos para la salud laboral: desde la inclusión de los factores de riesgo en las evaluaciones, la adecuada formación e información al trabajador/a, el establecimiento de una política clara sobre el control empresarial a través de las nuevas tecnologías, la implantación de protocolos contra el estrés, el tecnoestrés y la tecnoadicción... Pero sin lugar a dudas, la medida de prevención de riesgos laborales por excelencia contra el tecnoestrés es la desconexión digital en cualquiera de sus vertientes, desde establecer unos periodos de descanso durante la propia jornada laboral, hasta respetar los tiempos de descanso del trabajador. Su regulación legal, prevista en el art. 88 de la Ley Orgánica 3/2018, de 5 de diciembre, de Protección de Datos Personales y garantía de los derechos digitales (LOPD, en adelante), es importada del ordenamiento jurídico francés ${ }^{6}$ que, en agosto de 2016, reconoce el derecho a la desco-

${ }^{6}$ LOI n. ${ }^{\circ} 2016-1088$ du 8 août 2016 relative au travail, à la modernisation du dialogue social et à la sécurisation des parcours professionnels, JORF n. ${ }^{\circ} 0184$ du 9 août 2016. 
nexión digital , así como el deber de negociar las modalidades de su pleno ejercicio por el trabajador. En la misma línea que su homónimo francés, el art. 88 LOPD, aunque reconoce el derecho a la desconexión digital ${ }^{7}$, encomienda a la negociación colectiva o, en su defecto, a lo acordado entre la empresa y los representantes de los trabajadores, las modalidades de su concreto ejercicio, abriéndose un debate sobre su «escaso contenido obligacional», al no establecerse ningún régimen subsidiario en defecto de acuerdo (Moreno Vida, 2019: 177), puesto que "por muy potente que sea nuestra estructura de la negociación colectiva, siempre existirán vacíos de cobertura» (Cruz Villalón, 2019: 23).

Con todo, debe seguir incidiéndose en que, pese a la constante evolución en la protección de los riesgos psicosociales, la normativa de Seguridad y Salud Laboral sigue centrándose fundamentalmente en la prevención de los accidentes de trabajo y las enfermedades profesionales, puesto que se elaboró en función del modelo productivo tradicional (Fernández Avilés et al., 2018: 134). Es por ello que los instrumentos tradicionales del ámbito de la prevención de riesgos laborales no son suficientes para hacer frente a los nuevos riesgos propiciados a raíz de la incorporación de las NTIC al trabajo. La normativa habría de evolucionar desde una perspectiva meramente protectora hacia otra centrada en la promoción del bienestar de las personas trabajadoras (Fernández Martínez, 2019).

No obstante, uno de los mayores escollos a los que se ha de hacer frente en la prevención de riesgos laborales en la era digital es, precisamente, la calificación jurídica de las prestaciones de servicios a través de las NTIC como las plataformas digitales, la huida del Derecho del Trabajo a la que se ha hecho referencia como una de las características de la gig-economy. El nivel de protección en materia de seguridad y salud del trabajo autónomo es notoriamente inferior respecto del grado de protección reconocido a las personas que trabajan por cuenta ajena (López Rodríguez, 2019). Y es que pese a las previsiones del art. 8 y la disposición adicional duodécima de la LETA, dicha Ley no recoge expresamente los deberes relativos a la evaluación de riesgos, a la planificación de la prevención o a la gestión de la misma, obligaciones que, sin embargo, son esenciales para llevar a cabo auténtica labor de prevención de riesgos laborales (Rodríguez Egio, 2018: 517). Además, la calificación jurídica de los prestadores de servicios como trabajo por cuenta ajena conlleva la operatividad de la Ley 31/1995, de 8 de noviembre, de Prevención de Riesgos Laborales (LPRL, en adelante) ${ }^{8}$ y de su normativa de desarrollo, debiendo asumir el empresario la obligación de prote-

7 Sobre la regulación francesa y la italiana vid. Taléns Visconti, E.E. (2018): «La desconexión digital en el ámbito laboral: un deber empresarial y una nueva oportunidad de cambio para la negociación colectiva», Revista de Información Laboral, núm. 4, (BIB 201818599).

8 Debe tenerse en cuenta que la LPRL alude explícitamente a los trabajadores autónomos en dos preceptos: el art. 15.5 en referencia a la posibilidad de concertar operaciones de seguro con el fin de garantizar como ámbito de cobertura la previsión de riesgos derivados del trabajo; y el art. 24 para establecer los derechos y deberes en materia de coordinación de actividades empresariales. 
ger eficazmente a los trabajadores de los riesgos laborales, pudiendo afirmarse que, en la actualidad, sigue sin existir en el ordenamiento jurídico español un marco normativo suficiente que garantice a los trabajadores y trabajadoras autónomas una protección adecuada de la seguridad y salud en el trabajo (Fernández Avilés et al., 2018: 109).

\section{El papel de la negociación colectiva en la prevención de riesgos laborales en la industria digitalizada. La desconexión digital}

El III Acuerdo para el Empleo y la Negociación Colectiva 2015, 2016 y 2017 (BOE 20/06/2015), prorrogado por el IV Acuerdo para el Empleo y la Negociación Colectiva (BOE 18/07/2018), con el objeto de impulsar el empleo de calidad y con derechos, propugna que los convenios colectivos contemplen, entre otros objetivos fundamentales, la incidencia de las tecnologías de la información y de la comunicación en el desarrollo productivo general y en las relaciones laborales y en materia de seguridad y salud en el trabajo.

Sin perjuicio de que, como ya se ha puesto de relieve una y otra vez, las nuevas formas de trabajo enmarcadas en la gig-economy pretendan quedar fuera del ámbito de aplicación del Derecho del Trabajo, e incluso del difícil encaje de estos modelos de negocio en la vigente articulación de la normativa reguladora de la acción colectiva (Guerrero Vizuete, 2019: 274), el doble carácter de los instrumentos negociales, como fruto de la autonomía colectiva de las partes, pero con eficacia jurídica, otorga a la negociación colectiva un papel privilegiado a la hora de "adaptar» a las características de cada empresa o sector las emergentes necesidades en materia de seguridad y salud laboral derivadas de la incorporación de las NTIC. Se arguyen, entre otros muchos motivos (Meléndez MorilloVelarde, 2016): su función como norma reguladora de las condiciones laborales en un ámbito concreto; su capacidad para la adaptación de las condiciones generales de seguridad y salud en el trabajo a las características del sector de la producción o de la organización empresarial en el que será de aplicación a través de quienes mejor conocen las necesidades preventivas en ese ámbito; y su carácter temporal, que permite su actualización en referencia a los cambios normativos, a los acontecidos en el propio sistema productivo, e incluso a la aparición de nuevos riesgos producidos como consecuencia de esas novedades operadas en el tradicional sistema de producción.

Son muchos los aspectos en los que la negociación colectiva está llamada a mejorar las condiciones de seguridad y salud de los trabajadores (Aguilar Martín, 2017: 255 y ss.): vigilancia de la salud (regulando el controvertido tema de la voluntariedad de los reconocimientos médicos, las posibles sanciones a este respecto, o las pruebas concretas que han de realizarse según el tipo de activi- 
dad...); formación (por ejemplo, definiendo qué ha de entenderse por formación suficiente y adecuada según el puesto de trabajo); participación de los trabajadores (delimitando un modelo que facilite la "prevención participada»); información y consulta a los trabajadores (propiciando a través de este medio una política de comunicación interna definida y con objetivos concretos que incluyan los temas de salud); promoción de la salud (las posibilidades en este campo son ilimitadas, ya que tendrían cabida todo tipo de cláusulas que contribuyan a la promoción de un estilo de vida saludable en su concepción más amplia); y desde la entrada en vigor de la LOPD —e incluso antes- la desconexión digital.

Del estudio de la negociación colectiva en materia de prevención de riesgos laborales durante los primeros años de la década del siglo XxI (2000-2010), se concluye que no son muchos los convenios colectivos que regulan la prevención de riesgos laborales, y que la mayoría, tan sólo se limitan a realizar una mera remisión o reproducción literal de la normativa vigente, así como a enunciar compromisos genéricos de carácter programático, renunciándose a acometer medidas singulares que conviertan esas declaraciones de intenciones en actuaciones concretas, y obviándose la labor de concreción de las obligaciones genéricas impuestas al empresario en la normativa (Meléndez Morillo-Velarde, et al., 2011: 288 y ss.). Una situación que poco dista de la actual, en la que si bien ha aumentado considerablemente el número de convenios que incluyen alguna cláusula referida a la prevención de riesgos laborales, siguen sin hallarse muchas referencias negociales en materia de seguridad y salud laboral que vayan más allá de una simple declaración de principios, o de una reiteración de lo establecido en la Ley de Prevención de Riesgos Laborales que, en muchos casos, en una labor de síntesis mal entendida por los sujetos negociadores, aportan más bien confusión ${ }^{9}$.

En ese mismo estudio, referido a los ańos 2000-2010, también se concluye que las previsiones en el ámbito de la prevención de riesgos laborales sobre la introducción de nuevas tecnologías en el proceso productivo son muy escasas, sumamente genéricas, y que están orientadas exclusivamente a la necesidad de prestar una especial atención a tales cambios y de vigilar de un modo particular la salud de los trabajadores que presten sus servicios en terminales de ordenador (Meléndez Morillo-Velarde, et al., 2011: 289 y 290). Estudios más recientes aprecian una creciente intervención de los interlocutores sociales en la negociación en materia de prevención de los riesgos psicosociales, con especial atención a la prevención y tratamiento del acoso y de la violencia en el lugar de trabajo,

9 Una excepción es el Anexo I del CC Salas de Bingo de Alicante (BO. Alicante 10/05/2016), en el que se enuncian una serie de criterios y recomendaciones en materia de prevención de riesgos laborales provenientes del Proyecto IS-058/2004 financiado por la Fundación de Prevención de Riesgos Laborales, realizado por la Confederación Española de Juego (CEJ) y los Sindicatos UGT y Comisiones Obreras. 
pero siguen demandando una mayor atención a la prevención de los riesgos psicosociales vinculados a las nuevas tecnologías (Meléndez Morillo-Velarde, 2016).

Así pues, partiendo del hecho constatado de que la negociación colectiva no ha llegado a explotar las múltiples posibilidades que le ofrece el sistema en materia de prevención de riesgos laborales y las nuevas tecnologías, sin ánimo de exhaustividad, pero incidiendo en la carestía de convenios colectivos que atienden de un modo concreto la seguridad y salud laboral en relación con el empleo de las NTIC, así como en la incipiente — y cada vez más frecuente- regulación de la desconexión digital, a continuación van a reseñarse unos instrumentos negociales que pueden servir de ejemplo de la práctica negocial en esta materia.

Hay convenios, como el VIII Convenio colectivo estatal del corcho ${ }^{10}$, que simplemente se limitan a recoger la obligación de información y consulta a los trabajadores «relativa a la planificación y la organización del trabajo en la empresa y la introducción de nuevas tecnologías, en todo lo relacionado con las consecuencias que éstas pudieran tener para la seguridad y la salud de los trabajadores, derivadas de la elección de los equipos, la determinación y la adecuación de las condiciones de trabajo y el impacto de los factores ambientales en el trabajo» (art. 114).

Mención especial merece el XIX Convenio colectivo general de la industria química ${ }^{11}$, que prevé, entre otras medidas, la necesidad de que cualquier ampliación o modificación del proceso productivo que implique la incorporación de nuevas tecnologías respete el principio de mejora de la salud y seguridad de los trabajadores, y que cuando se implante una nueva tecnología se apliquen las técnicas de protección oportunas. Asimismo, establece «como prioritarias la promoción e intensificación de acciones organizativas, formativas e informativas de signo prevencionista que permitan al personal acomodarse a los cambios organizativos que las nuevas tecnologías puedan traer consigo preservando su salud física, mental y social, entendida como el concepto integral formulado por la Organización Mundial de la Salud», y prescribe la obligación de información y consulta al Comité de Seguridad y Salud Laboral, «respecto de todas aquellas decisiones relativas a la tecnología y organización del trabajo que tengan repercusión sobre la salud física y mental del trabajador, con carácter previo a su implantación».

Entre los pocos convenios colectivos que de alguna manera regulan aspectos de la prevención de riesgos laborales propios de la digitalización, puede reseñarse el Convenio colectivo para la industria fotográfica $(2017-2018-2019)^{12}$,

\footnotetext{
10 BOE 14/05/2020.

11 BOE 08/08/2018.

12 BOE 22/08/2017.
} 
cuyo art. 15.4 señala que «el trabajo de terminales de ordenador, pantallas de grabación y pantallas de vídeo, conlleva unas características que pueden derivar en situaciones de estrés, y otras enfermedades laborales. Para lo cual los trabajadores que prestan sus servicios en cualquiera de estos puestos de trabajo deberán tener la posibilidad de un sistema de organización del trabajo que les permita poder intercalar sus diferentes funciones con el fin de no permanecer demasiado tiempo continuo frente a la pantalla, facilitando así la disminución del riesgo a los trabajadores. Además, los trabajadores que realicen trabajos en este tipo de puestos pasarán una revisión médica especialmente concebida para el puesto que desempeñan (Oftalmología, Traumatología, etc.) que se realizará como mínimo cada ańo y correrá a cargo de la empresa». A cuyo tenor literal, el art. 76 del Convenio colectivo nacional de prensa no diaria ${ }^{13}$ añade, con muy buen criterio, un descanso de quince minutos, por cada dos horas de trabajo en videoterminales o pantallas de grabación. Un descanso que no podrá ser acumulado y que en todo caso tendrá la consideración de trabajo efectivo para el cómputo de la jornada, si bien, se admite la posibilidad de que durante este período se puedan desempeñar otras tareas siempre que estén dentro de su competencia. En la misma línea, el VII Convenio colectivo de Diario ABC, SL ${ }^{14}$, en el art. 61 señala que «La Dirección de la Empresa procurará que la organización del trabajo en todos los puestos con videoterminales esté programada de tal manera que permita sustituir la actividad en el videoterminal por otra distinta dentro de su trabajo", pero a diferencia de lo previsto en el Convenio colectivo nacional de prensa no diara, el periodo de descanso previsto es de diez minutos dentro de cada hora de trabajo efectivo ante dicho videoterminal, y sólo se disfrutará en aquellos supuestos en los que la organización del trabajo impida la realización de actividades alternativas al trabajo ante el videoterminal. Asimismo, en su art. 60, el VII Convenio colectivo de Diario ABC, SL dispone que el Comité de Seguridad y Salud en el trabajo, conforme al art. 18.2 LPRL respecto a los derechos de información, consulta y participación de los trabajadores, y al art. 39.2.b), realizará «con una periodicidad semestral visitas y revisiones de los puestos de trabajo en videoterminales o que revistan un especial riesgo auditivo en sus respectivos centros de trabajo, de cara a proponer en su caso las medidas correctoras adecuadas».

Por su parte, el art. 58 II Convenio colectivo estatal del sector de contact center (antes telemarketing) ${ }^{15}$ establece un listado mínimo de factores de riesgo que habrán de considerarse en la evaluación de riesgos y entre los que contempla los «Factores ergonómicos: aplicación de la Guía técnica del Instituto Nacional de Seguridad e Higiene para la evaluación de puestos de trabajo con pantallas de

\footnotetext{
13 BOE 23/12/2013.

14 BOE 27/02/2020.

15 BOE 12/7/2017.
} 
visualización; orden y limpieza; esfuerzos físicos que conllevan fatiga; listados de ordenador y documentos con caracteres de dimensión insuficiente y con espacios entre renglones insuficiente» y los «Factores psicosociales y de organización: pausas en el trabajo; tiempo entre llamadas inferior a 23/35 segundos en puestos de remarcación automática; fatiga y efectos negativos asociados por exigencias de la tarea de tipo físico y mental; conocimiento y claridad de los procedimientos de trabajo y su supervisión; conocimiento y claridad de las pautas a seguir en la gestión requerida por el cliente; horarios y turnos de trabajo que interfieran negativamente en la vida familiar». Y su art. 57 entre las medidas mínimas de vigilancia de la salud alude a la «Aplicación del protocolo de reconocimientos médicos para personas usuarias de pantallas de visualización del Ministerio de Sanidad, con especial valoración de los riesgos que puedan afectar a trabajadoras en situación de embarazo o parto reciente, y personas especialmente sensibles a determinados riesgos. (Cuestionario de función visual; reconocimiento oftalmológico; cuestionario de síntomas osteomusculares; examen del sistema osteomuscular; cuestionario de características de la tarea; cuestionario de la valoración de la carga mental)».

Como puede apreciarse en los ejemplos de negociación colectiva aludidos, la mayoría, en sintonía con lo expresado por Mella Méndez sobre las posibilidades de la negociación colectiva a este respecto, buscan dar cumplimiento al principio de la "prevención participada» en la empresa - previsto en la normativa de seguridad y salud laboral_-, o garantizar la información, consulta y participación de los trabajadores/as ante los cambios tecnológicos y organizativos, con el objeto de garantizar la transparencia e implicación del personal para el eficaz y seguro funcionamiento e implementación de los mismos (Mella Méndez, 2020). Sin embargo, quedan por explorar otras muchas opciones, fundamentalmente en el marco de la vigilancia de la salud, como la previsión de pruebas específicas atendiendo a los riesgos generados por el empleo de las NTIC para el desarrollo de la prestación laboral, y, sobre todo, de la formación en el uso de las NITC con el objeto de evitar el tecnoestrés y sus derivados. Por el contrario, sí que hay un tema de la prevención de riesgos laborales derivados del uso de las NTIC cuya regulación está en alza, y es el de la desconexión digital.

La desconexión digital es una obligación empresarial, que para el empleado no sólo ha de traducirse en un derecho, también en un deber, evitando así el "vampirismo adictivo" a la tecnología en tiempo de descanso por motivos "profesionales», el workaholics (Purcalla Bonilla, 2019). Aunque, como se verá, no está claro que en el ordenamiento jurídico español haya sido así concebido.

Antes incluso de su reconocimiento legal en España, ya el Convenio colectivo del Grupo Axa ${ }^{16}$, en su art. 14, tras una especie de exposición de motivos

16 BOE 10/10/2017. 
sobre la incidencia de los cambios tecnológicos y el fenómeno de la «interconectividad digital», reconoce el derecho de los trabajadores de AXA a no responder los emails o mensajes profesionales fuera de su horario de trabajo, salvo causa de fuerza mayor o circunstancias excepcionales ${ }^{17}$. Del mismo modo, el xvi Convenio colectivo de la ONCE y su personal ${ }^{18}$, en su anexo III, y como parte del plan de igualdad, entre las áreas de intervención para la consecución de los objetivos de dicho plan, dispone que «Las Partes valoran, como medida que promueve la efectiva conciliación de la vida personal, laboral y familiar, la denominada "desconexión digital» aplicada a herramientas de índole tecnológica e informática, como teléfonos y tablets con conexión de datos y ordenadores portátiles, compartiendo que su utilización no ha de ser motivo ni argumento para la extensión de la jornada laboral, ni para la interrupción de los tiempos de descanso o licencias de los trabajadores y trabajadoras». El art. 16.10 del Convenio colectivo de la empresa EUI Limited Sucursal España ${ }^{19}$, en el precepto dedicado a la jornada, con base en «la necesidad de poner límites entre el trabajo y la vida privada», pero «sin limitar las ventajas del trabajo flexible (FlexiTime) establecido para las áreas de soporte a negocio actuales y cualesquiera que puedan ponerse en marcha en el futuro", reconoce "el derecho de todos/as sus trabajadores/as a la desconexión digital y/o a cualquier tecnología de comunicación (teléfono, canales de mensajería, correos electrónicos, video-llamadas, y cualquier otro medio implantado en la empresa o al que estén acostumbrados los/as trabajadores/as) para garantizar el respeto al tiempo de descanso y/o vacaciones de todos/as sus trabajadores/as después del final de su jornada de trabajo». También el art. 65 del Convenio colectivo de la empresa Barcelona Cicle de L'Aigua S.A. $(B C A S A)^{20}$ se expresa en términos muy similares al resto de instrumentos negociales mencionados, pero a diferencia de éstos, su virtualidad reside en que el tratamiento del tema no se realiza exclusivamente desde la óptica de la conciliación de la vida personal, laboral y familiar sino, más ampliamente, desde la perspectiva de la seguridad y salud en el trabajo (Barrios Baudor, 2019).

17 «Los cambios tecnológicos producidos en las últimas décadas han provocado modificaciones estructurales en el ámbito de las relaciones laborales. Es innegable que hoy en día el fenómeno de la «interconectividad digital» está incidiendo en las formas de ejecución del trabajo mudando los escenarios de desenvolvimiento de las ocupaciones laborales hacia entornos externos a las clásicas unidades productivas: empresas, centros y puestos de trabajo. En este contexto, el lugar de la prestación laboral y el tiempo de trabajo, como típicos elementos configuradores del marco en el que se desempeña la actividad laboral, están diluyéndose en favor de una realidad más compleja en la que impera la conectividad permanente afectando, sin duda, al ámbito personal y familiar de los trabajadores y trabajadoras. Es por ello que las partes firmantes de este Convenio coinciden en la necesidad de impulsar el derecho a la desconexión digital una vez finalizada la jornada laboral. Consecuentemente, salvo causa de fuerza mayor o circunstancias excepcionales, AXA reconoce el derecho de los trabajadores a no responder a los mails o mensajes profesionales fuera de su horario de trabajo».

18 BOE $18 / 01 / 2018$.

19 BO de la Provincia de Sevilla 21/08/2018.

20 BO de la Provincia de Barcelona, 18/04/2018. 
Tras la entrada en vigor del art. 88 LOPD, en el que se reconoce el derecho a la desconexión digital, pero se encomienda a la negociación colectiva o, en su defecto, a lo acordado entre la empresa y los representantes de los trabajadores, las modalidades de su ejercicio, cada vez son más los convenios colectivos que pretenden hacer efectivo el derecho a la desconexión digital, pudiendo distinguirse, con carácter general, entre una regulación más genérica con ciertos resortes más programáticos que efectivos en el ámbito de sector y más precisa en el de empresa.

Los convenios colectivos de empresa, como los convenios colectivos Servicios Dix 2012, SL. ${ }^{21}$ y Grupo Selecta (AB Servicios Selecta España, SLU, Acorn Spain 1, SL y Servecave, SL) ${ }^{22}$, concretan el ejercicio del derecho a la desconexión digital como el derecho a no responder llamadas de teléfono, whastapp, correos... durante el tiempo de descanso, salvo fuerza mayor, o circunstancias excepcionales ligadas a necesidades de prestación de servicio a clientes. Algunos, como el art. 78 Convenio colectivo Carlson Wagonlit España, SLU ${ }^{23}$ especifican el espacio temporal de referencia como «el tiempo de descanso entre jornadas y de descanso semanal, así como en los períodos de vacaciones de los trabajadores o cualesquiera otros períodos de permisos retribuidos o días libres de conformidad con la legislación vigente», o como el art. 20 Convenio colectivo Servicios Dix 2012, SL. ${ }^{24}$, encomiendan a la comisión paritaria la vigilancia en la implantación de esta medida. Otros convenios, menos explícitos, únicamente prescriben un compromiso ad futurum, como por ejemplo el Convenio colectivo de Michelín España Portugal, SA, para los centros de trabajo de Tres Cantos (Madrid) y de Illescas (Toledo) ${ }^{25}$, cuyo art. 24, prescribe la obligación de crear durante la vigencia del convenio una Comisión "con el objeto de avanzar» en el ámbito de la desconexión digital. Más extensamente, el art. 21.3 del Convenio colectivo de Accepta Servicios Integrales, SLU ${ }^{26}$, tras afirmar que «la desconexión digital es un derecho cuya regulación contribuye a la salud de las personas trabajadoras disminuyendo, entre otros, la fatiga tecnológica o el estrés; y mejorando, de esta manera, el clima laboral y la calidad del trabajo» y que «la desconexión digital es además necesaria para hacer viable la conciliación de la vida personal y laboral, reforzando así las diferentes medidas reguladas en esta materia», reconoce el derecho y concede a las partes firmantes un plazo de 6 meses desde la entrada en vigor del convenio para el desarrollo «de los criterios para el ejercicio del derecho a la desconexión digital en los que, al menos, se especificarán las modalidades del ejercicio al derecho a la desconexión, especialmente

\footnotetext{
21 BOE 04/02/2020.

22 BOE 30/01/2020.

23 BOE 27/02/2020.

24 BOE 04/02/2020.

25 BOE 03/07/2020.

26 BOE 30/07/2020.
} 
en los supuestos de trabajo a distancia o en el domicilio particular» y regular el «derecho a la desconexión en aquellos otros casos en los que la prestación laboral exija disponibilidad o realización de guardias fuera del horario laboral establecido, así como de las personas que trabajan en plataformas digitales online, cuando existan estas formas de prestación laboral en las empresas del sector».

Las cláusulas negociales en el ámbito de sector suelen ser algo más inespecíficas que las de empresa. Tal es el caso del Anexo V del Convenio colectivo estatal de estaciones de servicio ${ }^{27}$, en el que viene a reiterarse lo dispuesto en el art. $88 \mathrm{LOPD}$, con alguna salvedad, como la advertencia de que las empresas no podrán tomar medidas sancionadoras contra quienes hagan uso efectivo de su derecho a la desconexión digital implantado en el seno de la empresa, tal y como hace el art. 48 del III Convenio colectivo estatal de la industria, la tecnología y los servicios del sector del metal (CEM) ${ }^{28}$, o la extensión del derecho a las personas que ocupen puestos directivos, como en el art. 16 del Convenio colectivo estatal de perfumería y afines ${ }^{29}$.

Especialmente amplia es la redacción del art. 89 del Convenio colectivo del Sector de Comercio Vario de la Comunidad de Madrid ${ }^{30}$, del art. 95 del Convenio colectivo estatal de centros y servicios veterinarios ${ }^{31}$, del protocolo de actuación para la desconexión digital del Convenio colectivo de Ahorramas, SA; Comercial Hermanos Vallejo, SA; Comercial Monte Igueldo, SL; Comercial Sierra, SL; J y M 44, SA; Majuan, SL; Monelja, SL; Rotterdam, SL y Rubio Martín, $\mathrm{SL}^{32}$, y en menor medida, del Anexo 9 del Convenio colectivo para las empresas del sector de harinas panificables y sémolas ${ }^{33}$. En líneas generales se sigue un mismo patrón, que puede ejemplificarse con el art. 95 del Convenio colectivo estatal de centros y servicios veterinarios ${ }^{34}$, que proclama el «derecho a la desconexión digital a fin de garantizar, fuera del tiempo de trabajo, el respeto de su tiempo de descanso, permisos y vacaciones, así como de su intimidad personal y familiar» y se compromete a la elaboración de una política interna «dirigida a las personas trabajadoras, incluidas los que ocupen puestos directivos, en la que definirán las modalidades de ejercicio del derecho a la desconexión y las acciones de formación y de sensibilización del personal sobre un uso razonable de las herramientas tecnológicas que evite el riesgo de fatiga informática», con particular atención a los supuestos de realización total o parcial del trabajo a distancia. Asimismo, se reconoce el derecho a «no responder a ninguna comunicación, fuere cual fuere

\footnotetext{
27 BOE $11 / 03 / 2020$.

28 BOE 19/12/2019.

29 BOE 20/08/2019.

30 BO. Comunidad de Madrid 26/10/2019.

31 BOE 14/08/2020.

32 BOE 01/08/2020.

33 BOE 17/06/2020.

34 BOE 14/08/2020.
} 
el medio digital utilizado, una vez finalizada su jornada laboral», con ciertas salvedades (periodos de disponibilidad del personal sanitario o circunstancias de fuerza mayor), y se señala que las empresas no podrán sancionar disciplinariamente a quienes ejerzan el derecho a la desconexión conforme a este precepto, además de que «el ejercicio del derecho a la desconexión digital no repercutirá negativamente en el desarrollo profesional de las personas trabajadoras».

Sin embargo, hay un aspecto claramente reseñable del art. 95 del Convenio colectivo estatal de centros y servicios veterinarios, que también puede hallarse en el protocolo de actuación para la desconexión digital del Convenio colectivo de Ahorramas, SA; Comercial Hermanos Vallejo, SA; Comercial Monte Igueldo, SL; Comercial Sierra, SL; J y M 44, SA; Majuan, SL; Monelja, SL; Rotterdam, SL y Rubio Martín, SL ${ }^{35}$, y es que el tenor literal de su apartado 2 del art. 95 determina que «Este convenio colectivo reconoce y formaliza el derecho a la desconexión digital como un derecho, pero no como una obligación. Esto implica expresamente que aquellas personas trabajadoras que quieran realizar comunicaciones fuera de su jornada laboral podrán hacerlo con total libertad». Una previsión que, de generalizarse en todos los instrumentos negociales, posiblemente terminaría desdibujando el derecho a la desconexión digital como medida de prevención de riesgos laborales.

Muy al contrario de estos casos en los que expresamente se advierte que el derecho a la desconexión digital no es una obligación. En otros países de nuestro entorno, como en Francia, los acuerdos sectoriales blindan la desconexión digital al reconocerla como una obligación. De hecho, determinadas empresas hacen efectiva dicha obligación, por ejemplo, con la instalación de una alerta para que cuando el trabajador/a haya alcanzado el límite de su jornada laboral, sea avisado/a del incumplimiento de su tiempo de descanso. Un caso singular es el de la empresa francesa Gie Réunica, donde el principal servidor de mensajería electrónica de la empresa bloquea los mensajes entrantes desde las 20:00 horas hasta las 07:00 horas del día laborable siguiente, y los fines de semana. De igual modo, en Alemania, la empresa Volkswagen bloquea el acceso a las comunicaciones corporativas desde el fin de la jornada laboral hasta el comienzo de la siguiente, y la empresa Daimler implementó un software que elimina los correos electrónicos recibidos durante las vacaciones y envía una respuesta automática informando de ello al emisor del correo e indicando la dirección de correo de otra persona que no está de vacaciones (Purcalla Bonilla, 2019). Medidas, todas ellas, que parecen quedar todavía bastante alejadas de los compromisos nacionales, donde el derecho a la desconexión digital es incipiente, el sector productivo no ha alcanzado un importante grado de tecnologización y el "presencialismo» forma parte de nuestra cultura laboral.

35 BOE 01/08/2020. 
Por último, cabe recordar que la desconexión digital puede ser regulada a través de la negociación colectiva, desde cualquier ámbito convencional incluidos los acuerdos interconfederales (nacionales o autonómicos) ${ }^{36}$, y en su defecto, por lo acordado entre la empresa y los representantes de los trabajadores, si bien, este trabajo se circunscribe, como su título indica, a la negociación colectiva en la prevención de riesgos laborales.

\section{Conclusiones y propuestas}

1. Pese a que la negociación colectiva sigue siendo el instrumento adecuado para regir las condiciones laborales en el marco de la industria 4.0, el primer hándicap con el que se enfrenta la negociación colectiva como garante de la seguridad y salud laboral de los trabajadores y trabajadoras en la prácticamente incipiente industria digital y en las empresas caracterizadas por la fusión de las tecnologías de la comunicación, el uso de internet y de las nuevas tecnologías, es el de la pretendida huida del Derecho del Trabajo característico de la gig-economy.

2. Las múltiples posibilidades que ofrece la negociación colectiva para concretar las obligaciones en materia de seguridad y salud no son aprovechadas por los sujetos negociadores. Rara vez se encuentran referencias negociales en materia de prevención de riesgos laborales que supongan algo más que una mera declaración de principios o una reiteración de lo ya previsto en la normativa, siendo mucho más insólito que un convenio colectivo regule aspectos relacionados con los riesgos laborales propios de la incorporación de las nuevas tecnologías al sistema de producción.

3. Los exiguos convenios que tratan medidas de prevención de riesgos laborales relacionadas con el uso de las NTIC suelen referirse al cumplimiento del principio de prevención participativa derivado de la incorporación de nuevas tecnologías, y los que ahondan algo más, provienen de ámbitos laborales en los que se trabaja en viodeterminales o pantallas de grabación.

4. Se echa en falta que los convenios colectivos aludan a los factores de riesgo propios del empleo de las NTIC en su concreto ámbito de aplicación, y a la necesidad de tenerlos en cuenta en las evaluaciones de riesgos y en la vigilancia de la salud, proponiendo, además, las pruebas y controles médicos destinadas a una detección precoz de cualquier patología relacionada con el uso de las NTIC.

5. Con el firme propósito de prevenir ciertas patologías propias del trabajo y su relación con las NTIC, sería conveniente que los convenios colectivos es-

36 Vid. por ejemplo, el Acuerdo Interprofesional de Cataluña para los años 2018-2020 (Diario Oficial de la Generalitat de Cataluña 07/09/2018). 
tablecieran una política clara sobre el control empresarial a través de las nuevas tecnologías, así como unos compromisos que definan una adecuada formación e información para los/as trabajadores/as en el uso de las NTIC para el desempeño de la prestación laboral. La formación e información en materia de prevención de riesgos laborales debe prestar especial atención a la correcta utilización de las NTIC y los instrumentos negociales podrían ser la fuente de dichos compromisos.

6. Al igual que la negociación colectiva se ha encargado de encomendar la formación de comisiones paritarias para el establecimiento de protocolos contra la violencia laboral, podría hacer lo propio con respecto a la creación e implantación de protocolos contra el estrés, el tecnoestrés y sus derivados: tecnoansiedad, tecnofatiga y tecnoadicción.

7. La negociación colectiva ha de hacer efectivo el derecho a la desconexión digital. A este respecto, los convenios colectivos de empresa han aprovechado para concretar determinados aspectos del mismo, mientras que los convenios colectivos de sector suelen limitarse a reproducir el tenor literal del art. 88 LOPD. Especialmente interesante es el compromiso a través de la negociación colectiva de garantizar acciones formativas y de sensibilización sobre el uso razonable de dispositivos digitales que evite el riesgo de fatiga informática.

8. Resulta cuestionable la configuración negocial del derecho a la desconexión digital con la advertencia de que se trata de un derecho, pero no una obligación, y que ello implica que los/as trabajadores/as que quieran realizar comunicaciones fuera de su jornada laboral podrán hacerlo libremente.

9. Sería ciertamente plausible que los sujetos negociadores blindaran la desconexión digital al reconocerla no sólo como un derecho, sino también como una obligación para los trabajadores y trabajadoras. En este sentido, se podría dar un paso más a través de cláusulas que dispongan el cierre automático de los servidores de correo una vez finalizada la jornada laboral o bloquearan el acceso a las comunicaciones corporativas desde el fin de la jornada laboral hasta el comienzo de la siguiente.

\section{Bibliografía}

Aguilar Martín, M. C. (2017): «El papel de la negociación colectiva en materia de seguridad y salud laboral. Una visión más amplia de la prevención", Revista Internacional y Comparada de Relaciones Laborales y Derecho del Empleo, Vol. 5, núm. 3, 2017, pp. 246-263.

Álvarez Cuesta, H. (2017): «La prevención de riesgos psicosociales en la economía colaborativa: los e-nómadas» en Fernández Domínguez, J.J. y Rodríguez EscanCIANo, S. (Dir.) VV.AA.: Tiempos de cambio y salud mental de los trabajadores, Bomarzo, pp. 55-82. 
Barrios Baudor, G. L. (2019): «El derecho a la desconexión digital en el ámbito laboral espańol: primeras aproximaciones», Revista Aranzadi Doctrinal, núm.1, (BIB 2018\14719).

Cruz Villalón, J. (2019): «Las facultades de control del empleador ante los cambios organizativos y tecnológicos», Temas Laborales. Revista Andaluza de Trabajo y Bienestar Social, núm. 150.

Fernández Avilés, J. A., Fernández Martínez, S., Mella Méndez, L., RodríguezRico Roldán, V. y Todolí Signes, A. (2018). El trabajo en la economía colaborativa y la prevención de riesgos laborales. Madrid: Secretaría de Salud Laboral y Medio Ambiente de UGT-CEC.

Fernández Avilés, J.A. (2017): «NTIC y riesgos psicosociales en el trabajo: estado de situación y propuestas de mejora", Diritto della Sicurezza sul Lavoro, Rivista dell'Osservatorio Olympus, núm. 2.

Fernández Martínez, S. (2019): «El impacto de la digitalización del mercado de trabajo en la salud mental de los trabajadores», Revista de Direito do Trabalho, edición especial CIELO Laboral.

García-Perrote Escartín, I. y Mercader Uguina, J. R. (2017): «La prestación de servicios en las plataformas digitales: los casos de BlaBlaCar y Take Eat Easy», Revista de Información Laboral, núm. 8, (BIB 2017\13002).

Ginés i Fabrellas A y Gálvez Durán S. (2016): «Sharing economy vs.uber economy y las fronteras del Derecho del Trabajo: la (des)protección de los trabajadores en el nuevo entorno digital», Indret, Revista para el análisis del Derecho, núm. 1, http:// www.indret.com/es/

GonzÁez Cobaleda, E. (2019): «Digitalización, factores y riesgos laborales: estado de situación y propuestas de mejora», Revista de Trabajo y Seguridad Social, CEF, número extraordinario.

Guerrero Vizuete, Esther (2019). «La digitalización del trabajo y su incidencia en los derechos colectivos de los trabajadores», Iuslabor, núm. 1.

Gutiérrez Arranz R. (2016): «Las relaciones laborales en la economía colaborativa: el caso Uber en EE.UU.», Nueva Revista Española de Derecho del Trabajo, núm. 187.

Hernández Bejarano, M. (2016): «El apoyo europeo al modelo de economía colaborativa: algunas cuestiones y propuestas para afrontar una regulación laboral y de seguridad social», Revista Española de Derecho del Trabajo, núm.192, (BIB 2016185594).

Jover Ramírez, C. (2018): «El fenómeno de la "gig economy» y su incidencia en el derecho del trabajo: aplicabilidad del ordenamiento jurídico laboral británico y español», Revista Española de Derecho del Trabajo, núm. 209, (BIB 201819632).

LAhera Fortaleza, J. (2019): "Las transformaciones del lugar de trabajo», Documentación Laboral, núm.118.

López Rodríguez, J. (2019): «La prevención de riesgos laborales en el trabajo a demanda vía aplicaciones digitales», Lan Harremanak, Revista de Relaciones Laborales, núm. 41.

Martín Muñoz, R. M.a (2020): «El ejercicio de los derechos colectivos en el entorno empresarial digital: representación y negociación colectiva de los trabajadores de plataformas digitales», Revista Española de Derecho del Trabajo, núm.232, (BIB 2020133794).

Meléndez Morillo-Velarde, L. (2016): «Nuevas tecnologías y riesgos psicosociales», Revista Española de Derecho del Trabajo, num.184, (BIB 20161704).

Meléndez Morillo-Velarde, L., Pérez Campos, A.I. y San Martín Mazzucconi, C. (2011): La prevención de riesgos laborales en la negociación colectiva. Un estudio com- 
parado de los años 2000 y 2010, Ministerio de Empleo y Seguridad Social, INSST, https:/www.insst.es/documents/94886/96076/negociacion+colectiva/e88a9faf-c22f4d6c-bf0e-32741c1a4956.

Mella Méndez, L. (2020): «Los retos de la prevención de riesgos laborales ante la digitalización de la empresa y las nuevas formas de trabajo: puntos críticos», Revista Española de Derecho del Trabajo, núm. 229 (BIB 2020\10658).

Mercader Uguina, J. R. (2017): El futuro del trabajo en la era de la digitalización y la robótica, Valencia, Tirant lo Blanch.

Mercader Uguina, J. R. (2018): "El mercado de trabajo y el empleo en un mundo digital», Revista de Información Laboral, núm.11, (BIB 2018\13994)

Moreno Vida, M. N. (2019): «Las facultades de control fuera de la jornada de trabajo: desconexión digital y control del trabajador», Temas Laborales. Revista Andaluza de Trabajo y Bienestar Social, núm. 150.

Purcalla Bonilla, M. A. (2019): "Control tecnológico de la prestación laboral y derecho a la desconexión de los empleados: Notas a propósito de la Ley 3/2018, de 5 de diciembre», Revista Española de Derecho del Trabajo, núm. 218, (BIB 201912891).

Rodríguez Egio, M. ${ }^{a}$ M. (2018): «Prevención de riesgos laborales en el ámbito de la economía colaborativa» en Alfonso Sánchez, R. y Valero Torrijos, J. (Dirs.): Retos jurídicos de la economía colaborativa en el contexto digital, Thomson Reuters Aranzadi.

RuESGA S.M. (2019): «El trabajo del futuro: más ocupación y menos jornada laboral», Revista de Derecho de la Seguridad Social Laborum. Economia y Sociología de la Seguridad Social y del Estado Social, núm. 21.

Sierra Benítez, E. M. (2015: «El tránsito de la dependencia industrial a la dependencia digital: ¿qué derecho del trabajo dependiente debemos construir para el siglo xxı?», Revista Internacional y Comparada de Relaciones Laborales y Derecho del Empleo, núm. 4, vol. 3.

TaléNs Visconti, E.E. (2018): «La desconexión digital en el ámbito laboral: un deber empresarial y una nueva oportunidad de cambio para la negociación colectiva", Revista de Información Laboral, núm. 4, (BIB 201818599).

Todolí Signes, A. (2017): El trabajo en la economía colaborativa, Valencia, Tirant lo Blanch.

Torrecilla García, J.A., Pardo Ferreira, C. y Rubio Romero, J.C. (2019): «Industria 4.0 y transformación digital: nuevas formas de organización del trabajo», Revista de Trabajo y Seguridad Social, CEF, número extraordinario.

Varela Ferrío, J. (2019): Digitalización de la empresa española. Panorámica de la realidad tecnológica del tejido productivo español, Documento elaborado por el servicio de estudios de la Confederación de UGT. 